\title{
Localization in multiple nearly-identical tuned vibration absorbers
}

\author{
Abdullah S. Alsuwaiyan \\ Department of Mechanical Engineering - Unaizah College of Engineering \\ Qassim University - Unaizah 51911 - Kingdom of Saudi Arabia \\ E-mail: asuwaiyan@qec.edu.sa, suwaiyana@yahoo.com
}

\begin{abstract}
The forced vibration of multiple nearly identical translational vibration absorbers is considered. Localization, where the amplitudes of vibration of a group of the absorbers can become relatively large as compared to those of the corresponding perfectly tuned system is investigated. In this work, imperfections amoung the absorbers are allowed and brought into the analysis through the stiffnesses of the absorbers' springs. Steady-state analysis of the mathematical model is used to obtain the results. Results, which focus on translational absorber systems, are found to be consistent with previous results obtained for torsional vibration absorbers. It is found that strengths of the localized responses depend on the levels of imperfections, the relative imperfections among absorbers, and the absorbers' damping. Localization is shown to exist in the undamped multiple nearly-identical vibration absorbers system, or more generally, in the system where the ratio of the level of absorbers' damping to the level of imperfections in the absorbers is small. It is also shown that localized responses can be effectively avoided by introducing some intentional mistuning in the absorbers' stiffnesses. This mistuning should be small in absolute magnitude in order not to reduce the absorbers' performance and should be large when compared to the absorbers' imperfections.
\end{abstract}

Keywords: Localization; Vibration Absorbers; Vibration Suppression; Imperfections; Mistuning.

\section{Introduction}

The greatest application of dynamic vibration absorbers is in synchronous machinery where absorber is tuned to a particular frequency and thus reduces or eliminates unwanted vibration. Absorbers are extensively used on large reciprocating internal combustion engines, which run at constant speeds for minimum fuel consumption. They are also used in many other applications where the operating frequency is nearly constant. In these apllications, the vibration absorber system balances the reciprocating forces and thus reduces vibration and its effects. In this paper, a system of multiple nearly identical vibration absorbers is considered. The phenomenon of vibration localization, where the response amplitudes of some of the absorbers can become realtively larger than those of the remaining absorbers is investigated.

In a nominally periodic structure, vibration localization occures when the couplings between the degrees of freedom of the structure are weak and there are some small imperfections among them. Localization makes the vibration energy to be concentrated in some confined regions of the structure. As a result, some components of the structure vibrate with small amplitudes, while some other components vibrate with significantly larger amplitudes. Anderson [1] was first to observed localization in the field of solid state physics. He showed that in a randomly ordered linear chain of particles, the wave function of the chain can exhibit spatially confined modes of motion.

One of the earliest studies of the phenomenon of localization in the field of structural dynamics was conducted by Hodges [2]. He showed that structural irregularities can result in localized motions in elastic systems. Localization in both linear and non linear dynamical systems has been studied by many researchers. Samples can be found in [3-8]. In a previous study of localization in systems of multiple vibration absorbers, Alsuwaiyan and Shaw [9] investigated the existence of localization in free vibration modes for both translational and torsional vibration absorber systems. They showed that the free vibration modes of these systems do localize when the ratio of the coupling between the absorbers to the imperfections among them is small. In another study [10], they investigated the existence of the forced vibration localization for the case when a system of multiple torsional vibration absorbers is under operation and subjected to a harmonic excitation. They showed that localization exists when there are some imperfections among the absorbers and absorbers' damping is small. 
In general, vibration localization is undesirable and its elimination is of interest to many researchers, see for example [11], [12] and refrences therein.

This work is aimed toward completing the picture of localization in multiple vibration absorber systems. A system of multiple nearly identical translational vibration absorbers under a harmonic excitation is considered. The existence of the forced vibration localization in such a system is investigated. The results of such a study are presented.

The paper is organized as follows: Section 2 describes the system of absorbers considered and the mathematical formulation. Section 3 describes the analytical expressions for the steady state response of the system. Section 4 presents the findings and a discussion. Section 5 introduces some sample results. Finally, the paper is closed with some conclusions in section 6 .

\section{Mathematical formulation}

A system of $N$ vibration absorbers mounted on a primary mass, $M$, as shown schematically in Figure 1 is considered. The equations of motion are

$M \ddot{q}+c \dot{q}+k q+\sum_{i=1}^{N}\left(k_{i} x_{i}+c_{i} \dot{x}_{i}\right)=F_{o} \sin (\omega t)$

$\ddot{x}_{i}+\mu_{i} \dot{x}_{i}+\omega_{i}^{2} x_{i}=\ddot{q}$,

where $x_{i}$ is the relative position of the $i$ th absorber, $i . e$., $x_{i}=q-z_{i}, \mu_{i}=c_{i} / m_{i}$, and $\omega_{i}^{2}=k_{i} / m_{i}$. Figure 1 clarifies the meaning of the other variables in the above equations. When equation(1) is solved for the acceleration of the primary mass, $\ddot{q}$, and then substituted into equation(2), the following equation is obtained:

$\ddot{x}_{i}+\mu \dot{x}_{i}+\omega_{i}^{2} x_{i}=\frac{m}{M}\left[f_{o} \sin (\omega t)-\frac{c}{m} \dot{q}-\frac{k}{m} q-\sum_{j=1}^{N}\left(\omega_{j}^{2} x_{j}+\mu \dot{x}_{j}\right)\right]$

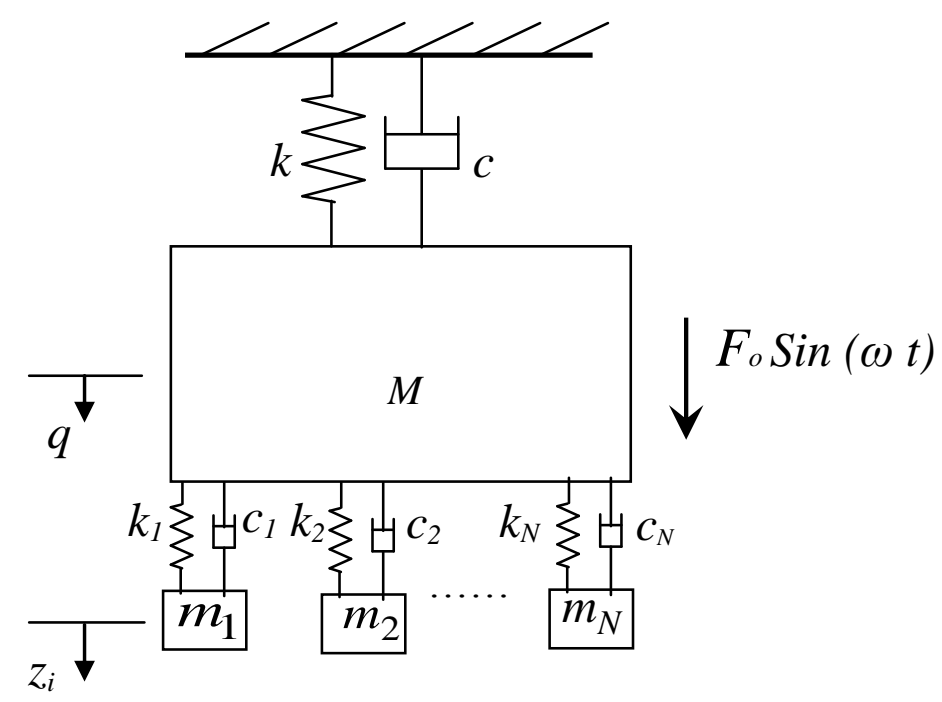

Fig. 1: $N$ absorbers mounted on a primary mass, $M$.

To reach to this equation, it is assumed that all absorbers have the same mass, $m$, and the same damping coefficient, $c_{a}$, i.e., $m_{i}=m, c_{i}=c_{a} \forall i \in[1, N]$. The quantities $f_{o}$ and $\mu$ are $F_{o} / m$ and $c_{a} / m$ respectively.

The absorbers are usually tuned to the frequency of the applied load. However, in this analysis small mistunings in the absorbers' springs stiffnesses are allowed, i.e.,

$k_{i}=k_{n}\left(1+\delta_{i}\right)$,

where $k_{n}$ is the nominal spring stiffness which corresponds to the perfectly tuned system, i.e., $\omega^{2}=k_{n} / m$, and $\delta_{i}$ represents a measure of the mistuning of the $i$ th absorber. This leads to the following relationship between the natural frequency of the $i$ th absorber and the frequency of the applied load: 
$\omega_{i}^{2}=\omega^{2}\left(1+\delta_{i}\right)$

Since this analysis is limited to small mistunings, it is expected that the primary mass will be near stationary at steady state. According to this and to simplify the analysis, it is convinient to ignore the terms $\left(\frac{c}{m} \dot{q}\right)$ and $\left(\frac{k}{m} q\right)$ in equation(3), (it should also be noted that these terms are multiplied by $m / M$ which is usually small). Rearranging equation(3), and ignoring small terms yields

$\ddot{x}_{i}+\omega^{2} x_{i}=-\omega^{2} \delta_{i} x_{i}-\mu \dot{x}_{i}+r\left[f_{o} \sin (\omega t)-\sum_{k=1}^{N} \omega^{2} x_{k}\right]$,

where $r$ is the mass ratio, i.e., $r=m / M$.

The conclusions drawn from the steady-state response of this system of equations, which is considered in the following section, represent the main results of this work.

\section{The steady state analysis}

To formulate the problem so that the absorbers' steady state responses can be found, it is assumed that $x_{i}=x_{i}^{s S} e^{j \omega t}$, where $j=\sqrt{-1}$, and $x_{i}^{s S} \in C$. When this is substituted in equations(6), the following system of equations (in matrix form) is obtained:

$(\boldsymbol{A}+\boldsymbol{B}) \boldsymbol{X}^{s S}=\boldsymbol{f}$

where

$\boldsymbol{A}=\operatorname{Diag}_{N}\left(\delta_{i}+j \frac{\mu}{\omega}\right), \quad \boldsymbol{B}=r \operatorname{Ones}(N)$

$\boldsymbol{X}^{s S}=\left(\begin{array}{c}x_{1}^{S S} \\ \vdots \\ x_{N}^{S S}\end{array}\right)$, and $\boldsymbol{f}=\left(r / \omega^{2}\right) f_{o}$ One $(N)$.

$\operatorname{Diag}_{N}\left(\mathrm{~s}_{\mathrm{i}}\right)$ denotes an $N$ by $N$ diagonal matrix with $s_{i}$ in the $i$ th diagonal entry, $\operatorname{Ones}(N)$ denotes an $N$ by $N$ matrix with every element equal to one, and $O n e(N)$ denotes an $N$ by 1 vector with every element equal to one.

Uncoupling this system yields the following equation for the $i$ th absorber:

$\left(R_{i}+j I_{i}\right) x_{i}^{s s}=f$

where

$R_{i}=\left[\delta_{i}+r \sum_{k=1}^{N}\left(\delta_{i} \delta_{k}+\left(\frac{\mu}{\omega}\right)^{2}\right) /\left(\delta_{k}^{2}+\left(\frac{\mu}{\omega}\right)^{2}\right)\right]$,

$I_{i}=\frac{\mu}{\omega}\left[1+r \sum_{k=1}^{N}\left(\delta_{k}-\delta_{i}\right) /\left(\delta_{k}^{2}+\left(\frac{\mu}{\omega}\right)^{2}\right)\right]$

and $f=\left(r / \omega^{2}\right) f_{o}$.

The steady-state vibration amplitude of the $i$ th absorber will then be given by

$X_{i}=\left|x_{i}^{S S}\right|=r f_{o} /\left(\omega^{2} \sqrt{\left(R_{i}\right)^{2}+\left(I_{i}\right)^{2}}\right)$.

\section{Discussion}

When damping in the absorbers is small compared to mistunings, the vibration amplitude given by equation(9) above can be approximated by

$X_{i} \approx r\left(f_{o} / \omega^{2}\right) /\left[\delta_{i}+r\left(1+\sum_{k=1, k \neq i}^{N} \delta_{i} / \delta_{k}\right)\right]$

Examining this equation, it can be seen that the amplitudes of vibration for all absorbers will be the same as long as the levels of mistuning are the same. However, due to the fact that there are always some imperfections among the absorbers and because of the presence of the ratio $\left(\delta_{i} / \delta_{k}\right)$, one should consider the possibility of the existence of some localized responses. Equation(10) indicates that when the mistuning levels of all absorbers are close to zero and a sub- 
group of absorbers have mistuning levels that are smaller than those of the remaining absorbers, then absorbers with the smaller mistuning levels will localize, meaning that their amplitudes of vibration will be higher than those of the remaining absorbers. The strengths of this localized responses depend on the magnitudes of the mistunings of the localizing sub-group and how far they are mistuned from the remaining absorbers.

If vibration absorbers are used, they are most often used without damping. This is due to the fact that damping defeats the ellimination of unwanted vibration. However, when damped absorbers are used and when damping is large compared to mistunings, localization will not occur.

It should be noted here that localization is expected to occur only when the levels of mistuning of all absorbers are close to zero and there are variations among them. This suggests that a solution to avoid localized responses is to introduce some identical intentional mistuning among the absorbers. If this intentional mistuning is positive, it will shift the frequency where localization takes place, $\sqrt{\left(k_{n}\left(1+\delta_{n}\right)\right) / m_{a}}$, to be after the absorbers operating frequency, $\sqrt{k_{n} / m_{a}}$. In the other hand, negative intentional mistuning will lower the frequency where localization occurs, $\sqrt{\left(k_{n}\left(1-\delta_{n}\right)\right) / m_{a}}$, which means that localized responses will take place before reaching the absorbers operating frequency. So, the intentional mistuning should be positive. It should also be noted that the intentional mistuning in the absorbers must be large compared to absorbers' imperfections and small in absolute magnitude in order not to affect the absorber's performance. It has long been known that intentional mistuning is useful for the case of torsional vibration absorber systems. In centrifugal pendulum vibration absorbers (CPVAs) with circular paths, intentional mistuning of the absorbers' paths, help to avoid nonlinear jump behavior [13] and other types of nonlinear instabilities [10].

The following numerical example will clarify these findings.

\section{$5 \quad$ Numerical example}

A system of four vibration absorbers mounted on a machine whose vibration is to be supressed is considered. The machine is modeled as a simple mass of $200 \mathrm{~kg}$ attached to ground through a spring with a stiffness of $30 \mathrm{kN} / \mathrm{m}$. The mass is subjected to a harmonic force of a frequency $10 \mathrm{rad} / \mathrm{s}$. Four absorbers are attached to the main mass and tuned to the frequency of exitation. Each absorber has a mass of $10 \mathrm{~kg}$, which means that the nominal spring stiffness, $k_{n}$, of each absorber is $1000 \mathrm{~N} / \mathrm{m}$.

Three cases are considered. In the first case, all absorbers have some small imperfections in their stiffnesses and one of them has a smaller imperfection than the remaining three (Table 1). In the second case, two absorbers have smaller imperfections than the other two (Table 2). In the third case, imperfections among absorbers are the same as those in cases 1 and 2, but a nominal intentional mistuning level of 3\% (small in absolute magnitude but large comared to imperfections) is introduced in all absorbers' stiffnesses (Tables 3 and 4).

Table 1: Data for case 1

\begin{tabular}{|c|c|c|c|c|}
\hline Absorber & 1 & 2 & 3 & 4 \\
\hline$k_{i}$ & 1001 & 1010 & 1012 & 1008 \\
\hline$\delta_{i}(\%)$ & 0.1 & 1.0 & 1.2 & 0.8 \\
\hline
\end{tabular}

Table 2: Data for case 2

\begin{tabular}{|c|c|c|c|c|}
\hline Absorber & 1 & 2 & 3 & 4 \\
\hline$k_{i}$ & 1001 & 1002 & 1011 & 1012 \\
\hline$\delta_{i}(\%)$ & 0.1 & 0.2 & 1.1 & 1.2 \\
\hline
\end{tabular}

Table 3: Data for case 3-1

\begin{tabular}{|c|c|c|c|c|}
\hline Absorber & 1 & 2 & 3 & 4 \\
\hline$k_{i}$ & 1031 & 1040 & 1042 & 1038 \\
\hline$\delta_{i}(\%)$ & 3.1 & 4.0 & 4.2 & 3.8 \\
\hline
\end{tabular}

\begin{tabular}{|c|c|c|c|c|}
\hline \multicolumn{7}{|c|}{ Table 4: Data for case 3-2 } \\
\hline Absorber & 1 & 2 & 3 & 4 \\
\hline$k_{i}$ & 1031 & 1032 & 1041 & 1042 \\
\hline$\delta_{i}(\%)$ & 3.1 & 3.2 & 4.1 & 4.2 \\
\hline
\end{tabular}

Equation (10) is used to find the normalized amplitude of vibration of each absorber. To check the accuracy of the results, amplitudes obtained from Equation (10) are compared to those obtained from the steady state solution of the complete system (i.e. equations (1),(2)). 
Figure 2 shows that localization indeed exists for the undamped absorbers. In case 1, the amplitude of vibration of absorber no. 1 is higher than the remaining three since it has a smaller imperfection. In case 2, the absorbers with smaller imperfections (i.e. absorbers 1 and 2) are localizing. It is also clear from Figure 1 that localization strength depends on both the variations among the absorber tunings and the number of absorbers with significantly different mistunings. One can also see the good agreement between the theory, i.e. Equation (10), and the steady state solution of the complete system.

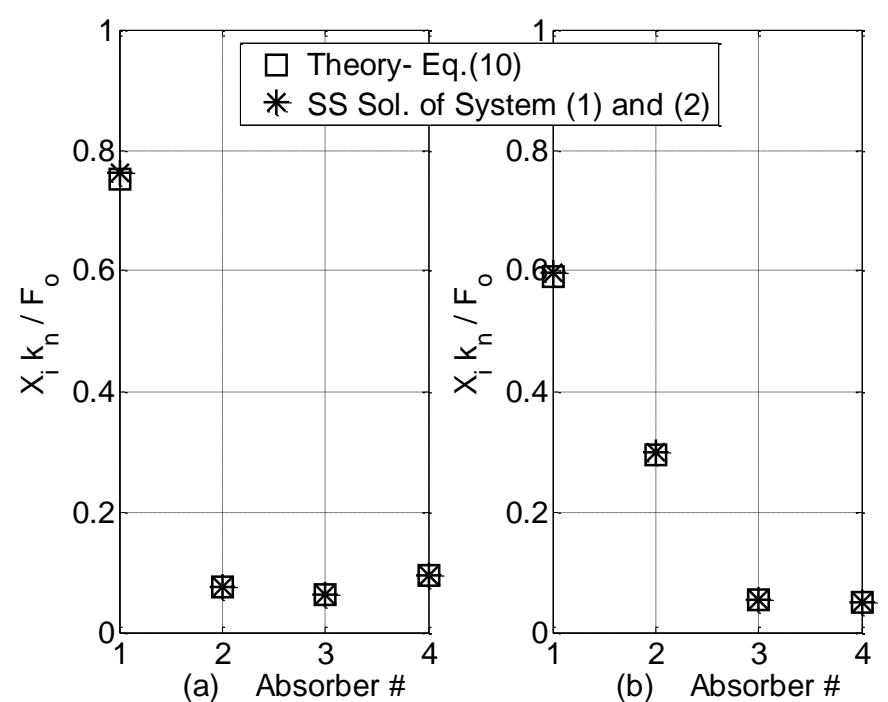

Fig. 2: Absorbers' normalized relative amplitudes, (a) case 1, (b) case 2.

Figure 3 shows that in the presense of some nominal intentional mistuning (small in absolute magnitude but large when compared to imperfections), localization is avoided eventhough the relative impefections among absorbers is simillar to those in cases 1 and 2, (i.e. one absorber has a smaller imperfection than the remaining three and two absorbers have smaller imperfections than the other two). Again, good agreement between the theory and the steady state solution of the complete system can be seen.

Figure 4 shows a plot of the vibration amplitudes for absorbers with imperfections similar to those in case 1 for the three absorbers that are not localizing (absorbers $2,3,4$ ) and varying the imperfection of absorber 1 . It is clear from this Figure that localization is stronger when the imperfection of absorber 1 is smaller than those of the other absorbers. It is interesting to notice that when absorber 1 has no imperfection, i.e. absorber 1 is perfectly tuned to the exitation frequency, localization will be maximum and absorber 1 will take care of the whole vibration absorption process keeping all other absorbers near stationary. On the other hand, when its imperfection gets closer to those of other absorbers, localization gets weaker.

In addition, localization strength depends on the forcing frequency as shown on Figure 5 (a). When the forcing frequecy gets closer to the nominal absorbers frequency, localization becomes stronger. Figure 5 (b) shows that the introduction of intentional mistuning takes care of localization for all frequencies up to the absorbers's nominal frequency.

Figure 6 shows the effect of both localization (case 1) and 1\% intentional mistuning (case 3-1) on the absorbers' performance. It is clear from this Figure that these effects are small and can be ignored. this is true as long as imperfections in absorbers stiffnesses are small and close to zero and the intentional mistuning is also small in absolute value. This means that designers need to pay attention to localization only when large vibration amplitudes of absorbers is to be restricted due to space limitation or any other reason.

It should be noted that localization can also be avoided by introducing some damping to the absorbers. However, damping should be kept as small as possible since it affects the absorbers' performance. But, if the absorbers application requires introducing some moderate damping level to the system, then one needs not to worry about localization since damping will take care of it. To show this, absorbers with two different damping levels, $\mu=0.1,0.5$, and imperfections similar to those in case 1 are considered. The absorbers' normalized relative amplitudes are shown in Figure 7. It is clear that as the damping level is increased, localization gats weaker until it disappear. 


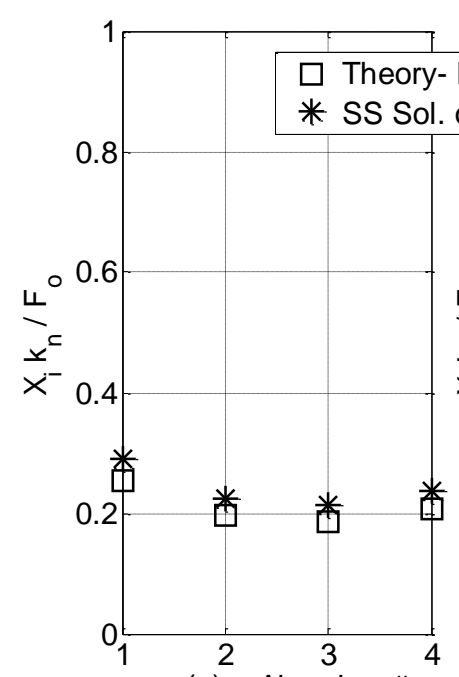

(a) Absorber \#

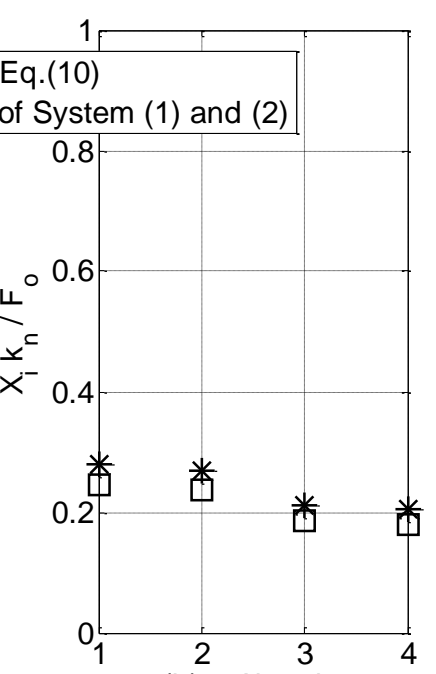

(b) Absorber \#

Fig. 3: Absorbers' normalized relative amplitudes, (a) case 3-1, (b) case 3-2.

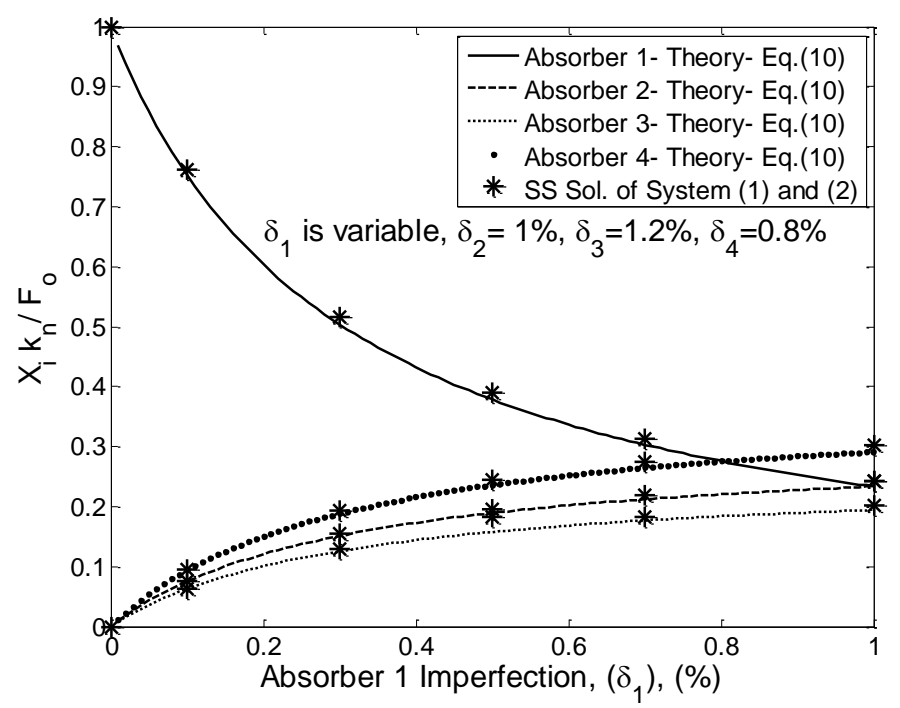

Fig. 4: Effect of varying the imperfection of absorber 1 on localization
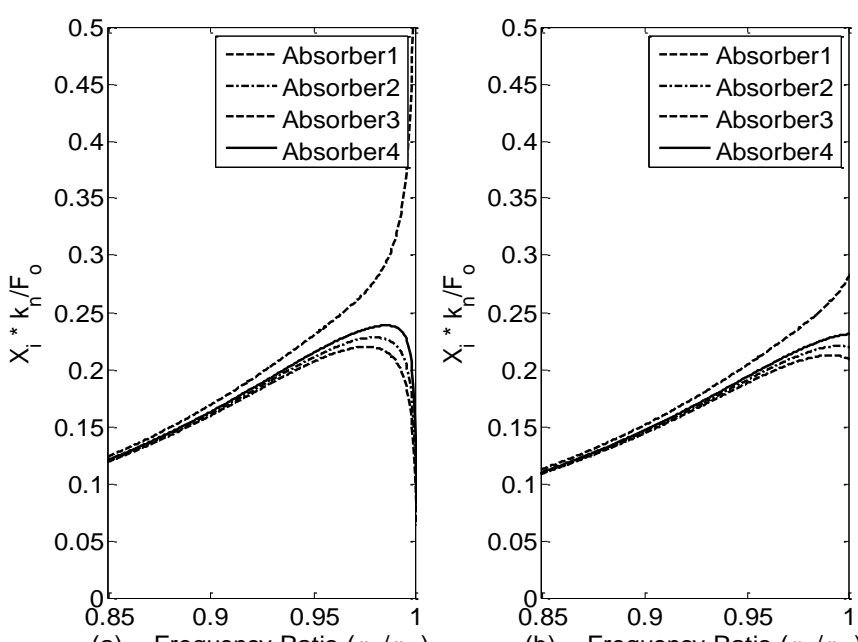

(a) Frequency Ratio $\left(\omega / \omega_{a}\right)$

(b) Frequency Ratio $\left(\omega / \omega_{a}\right)$

Fig. 5: Absorbers' normalized amplitudes as functions of frequency ratio, (a) case1, (b) case 3-1 


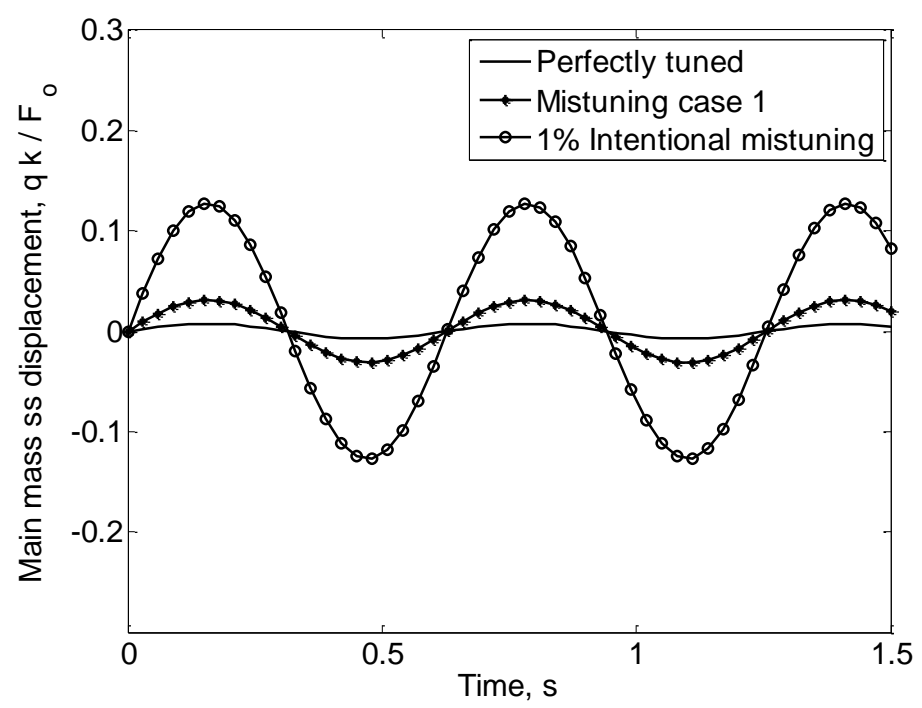

Fig. 6: Main mass normalized steady state displacement for different mistunings

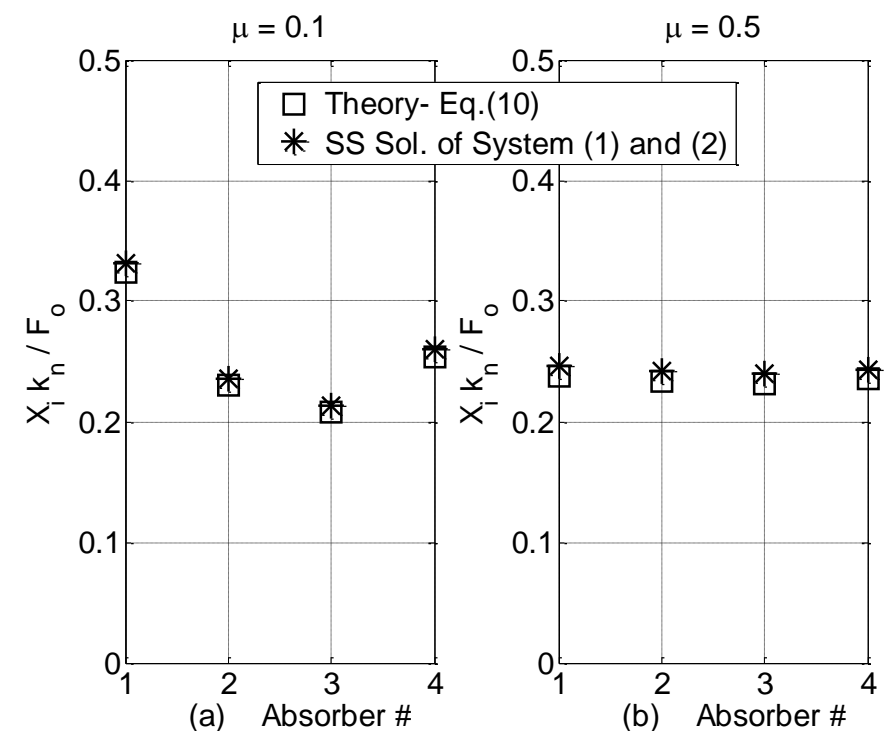

Fig. 7: Absorbers' normailzed relative amplitudes for different damping levels

\section{Conclusions}

In the presence of small imperfections between the tuned absorbers, localization exists in the forced steady-state response of vibration absorber systems. The relative strengths of the localized responses depend on (i) the variations among the absorber tunings (ii) the number of absorbers with significantly different mistuning and (iii) the ratio of the level of damping to the level of imperfections. Systems with higher differences in imperfections are expected to have stronger localized responses, especially if only a small number of absorbers have a significant difference from their partners. Also, systems with lower damping to imperfection ratio are expected to have stronger localized responses.

An effective way to avoid localization in the undamped vibration absorber systems is to introduce a small, but large when compared to imperfections, positive intentional mistuning in the absorbers' springs. This renders those systems robust against localization, yet maintains good performance of the absorbers. This mistuning offers a wide range of benefits, including the avoidance of localization.

Localization can also be avoided by introducing a moderate level of damping in the absorbers. To successfully eliminate localization, damping level should be large when compared to absorbers' imperfections and as small as possible in absolute value in order not to affect the absorbers effectiveness. 


\section{References}

[1] P. W. Anderson," Absence of diffusion in certain random lattices", Physical Review 109 (1958), pp 1492-1505.

[2] C. H. Hodges," Confinement of vibration by structural irregularity", Journal of Sound and Vibration 82 (1982), pp 411-424.

[3] Divyendu Sharma, S. S. Gupta, R. C. Batra," Mode localization in composite laminates”, Composite Structures 94 (2012), pp $2620-26431$.

[4] Marcel F. and Svitlana M," Strong localization induced by one clamped point in thin plate vibrations", Physical Review letters 103 (2009), pp 254301/1-254301/4.

[5] Docekal A., Dynybyl V., Kreidl M., Samid R. and zak P.,” Localization of the best measuring point for gearwheel behaviour testing using group of adaptive models evolution", Measurement Science Review 8 - Section 1 No. 2 (2008), pp 42-45.

[6] M. E. King and P. A. Layne,” Dynamics of nonlinear cyclic systems with structural irregularity”, Nonlinear Dynamics 15 (1998), pp $225-244$.

[7] C. P. Chao and S. W. Shaw,"Nonlinear localization in systems of tautochronic vibration absorbers", Proceedings 1997 ASME Design Engineering Technical Conferences, paper DETC97/VIB-3956.

[8] A. F. Vakakis and T. K. Centikaya,"Mode localization in a class of multi-degree of freedom systems with cyclic symmetry", SIAM Journal on Applied Mathematics 53 (1993), pp 265-282.

[9] A. S. Alsuwaiyan and S. W. Shaw," Localization of free vibration modes in systems of nearly-identical vibration absorbers", Journal of Sound and Vibration 228(3) (1999), pp 703-711.

[10] A. S. Alsuwaiyan and S. W. Shaw," Steady-state responses in systems of nearly-identical torsional vibration absorbers", ASME Journal of Vibration and Acuistics 125 (2003), pp 80-87.

[11] S. M. Shahruz,"Elimination of vibration localization in mistuned periodic structures", Journal of Sound and Vibration 281 (2005), pp $452-462$.

[12] S. M. Shahruz,'Elimination of vibration localization: a mathematical justification", Journal of Sound and Vibration 283 (2005), pp 449-458.

[13] D. E. Newland," Nonlinear aspects of the performance of centrifugal pendulum vibration absorbers", ASME Journal of Engineering for Industry 86 (1964), pp 257-263. 